\title{
Attentional Bias Modification Training for Patients with Generalised Anxiety Disorder: a Randomised Controlled Study
}

\author{
SWH Chau, CY Tse, SHW So, SSM Chan
}

\begin{abstract}
Background: Hypervigilance to threat is a mechanism contributing to generalised anxiety disorder (GAD). Although attentional bias modification training (ABMT) is designed to reduce attention to threat, its use as a mechanistically focused psychological intervention for GAD has not been examined. We aimed to investigate the effect of a brief ABMT on reducing anxiety, worry, and attentional bias in outpatients with GAD, and to determine the association between change in attentional bias and changes in anxiety and worry.

Methods: This was a parallel-group, double-blind, randomised controlled trial. Patients with GAD who had no changes after medication treatment for the past 8 weeks were randomly allocated to either the treatment or control group to receive 4 weekly sessions of ABMT or sham ABMT, respectively, in addition to standard care. Anxiety was measured using the Hamilton Anxiety Rating Scale and the StateTrait Anxiety Inventory - trait anxiety subscale. Pathological worry was measured using the Penn State Worry Questionnaire. Attentional bias was measured using the bias score.

Results: A total of 33 participants were allocated to the treatment $(n=17)$ or control $(n=16)$ groups. Both groups reported a significant reduction in levels of anxiety and worry after intervention, but the reduction was not greater after ABMT than sham ABMT. There was no significant change in attentional bias after ABMT; change in attentional bias was not correlated to changes in anxiety and worry.

Conclusion: The efficacy of the brief ABMT as a mechanistically focused treatment for GAD was not supported. The small sample size and short duration of treatment may have rendered the results inconclusive.
\end{abstract}

Key words: Anxiety disorders; Attentional bias

Steven Wai Ho Chau, MBBS, MSc, MRCPsych, FHKAM(Psychiatry), Department of Psychiatry, Tai Po Hospital, Hong Kong

Chun-Yu Tse, MPhil, PhD, Department of Psychology, The Chinese University of Hong Kong, Hong Kong; Centre for Cognition and Brain Studies, The Chinese University of Hong Kong, Hong Kong

Suzanne Ho-wai So, MA, MSc, MSSc, PhD, Department of Psychiatry, The Chinese University of Hong Kong, Tai Po Hospital, Hong Kong

Sandra Sau Man Chan, MBChB, FRCPsych, FHKAM(Psychiatry), Department of Psychiatry, The Chinese University of Hong Kong, Tai Po Hospital, Hong Kong

Address for correspondence: Dr Steven Wai Ho Chau, Department of Psychiatry, l/F Tai Po Hospital, Chuen On Road, Tai Po, Hong Kong. Email: stevenwhchau@gmail.com

Submitted: 17 October 2017; Accepted: 9 May 2018

\section{Introduction}

Generalised anxiety disorder (GAD) is characterised by persistent and generalised worry and anxiety, together with somatic symptoms related to hypervigilance and physiological hyperarousal. ${ }^{1}$ GAD affects $4.5 \%$ to $5.7 \%$ of the general population, ${ }^{2}$ and imposes a high cost on society. ${ }^{3}$ Only $50 \%$ to $60 \%$ of patients are responsive to recommended medication. ${ }^{4,5}$ Therefore, alternative interventions to target cognitive processes that maintain GAD are suggested.

According to cognitive models of GAD, the enhanced registration of negative stimuli, activation of the primary threat appraisal system with hypervigilance and autonomic hyperarousal, and recruitment of excessive worry as the secondary appraisal strategy trigger and perpetuate anxiety states. ${ }^{6}$ Cognitive behavioural therapy and mindfulnessbased psychotherapy for GAD have been shown to be effective,${ }^{7,8}$ but they are not often accessible to patients.

Attentional bias modification training (ABMT) is a treatment approach that targets the earliest stage of the anxiety cascade. Attentional bias is systematic selectivity of attention allocation in favour of one type of information over another, ${ }^{9}$ and has been demonstrated in anxiety-prone and clinically anxious individuals. Using the visual probe task, patients with GAD have shown attentional bias towards threatening facial or lexical stimuli. ${ }^{10,11}$ Earlier experiments have demonstrated that attentional bias in non-clinical but anxiety-prone participants can be modified by ABMT, and that changes in attentional bias can lead to changes in vulnerability to stress. ${ }^{12,13}$

ABMT has achieved promising results in non-clinical populations. It has been suggested as an intervention for anxiety and GAD, because it is fully computerised and 
operator-independent and therefore potentially more accessible. Typically, ABMT involves a visual probe procedure, ${ }^{14}$ in which participants are trained to divert attention away from threatening stimuli through the topdown pathway via repeated implicit learning. ${ }^{15,16}$

However, the efficacy of ABMT as an intervention for anxiety is inconsistent in meta-analyses, ${ }^{17,18}$ and there is publication bias. Many ABMT studies are uncontrolled. Among controlled studies, most evidence for reduction in anxiety and attentional bias after ABMT involves nonclinical or sub-clinical samples only; these limitations in sampling and methodology affect the representativeness and generalisability of the results. ${ }^{19}$

In a meta-analysis study on the effect of ABMT on reducing anxiety among patients with anxiety disorders, ${ }^{20}$ only one of the included studies focused on patients with GAD and reported that ABMT achieved a stronger reduction in both attention bias (Cohen's $d=0.86$ ) and anxiety (Cohen's $d=0.72-73$ ). ${ }^{10}$ These preliminary findings supported the use of the 4-weekly ABMT in ameliorating anxiety, worry, and attention bias; however, that study was not reported in accordance with CONSORT guidelines and had limited characterisation of participants, and its results have not been replicated since.

The present study aimed to examine the efficacy of ABMT, in addition to usual psychiatric care, in treating patients with GAD. The main hypotheses are: (1) reduction in anxiety and worry is greater after ABMT than after sham ABMT; (2) reduction in attentional bias is greater after ABMT than after sham ABMT; and (3) change in attentional bias is associated with changes in anxiety and worry.

\section{Methods}

This parallel-group, double-blind, randomised controlled study was approved by the Joint Chinese University of Hong Kong-New Territories East Cluster Clinical Research Ethics Committee (Reference: 2014.382-T). It was registered in the trial registry of the Centre for Clinical Research and Biostatistics of the Chinese University of Hong Kong (Reference: CUHK_CCT00431). Written informed consent was obtained from each participant.

\section{Participants}

Patients were recruited from two psychiatric specialist outpatient clinics in Hong Kong (Alice Ho Miu Ling Nethersole Hospital and North District Hospital) from October 2014 to June 2015. Potential participants were identified by triage nurses, clinical psychologists, and psychiatrists and were screened by the first author. Inclusion criteria were (1) age 18 to 64 years, (2) diagnosis of GAD based on the Structured Clinical Interview for the DSM-IV, ${ }^{21}$ and (3) no changes after antidepressant or benzodiazepine regimen over the past 8 weeks. Exclusion criteria were (1) presence of suicide risk, (2) history of any psychotic disorder, bipolar affective disorder, and/or organic mental disorder, (3) alcohol or substance abuse, (4) inability to read traditional Chinese, and (5) currently receiving structured psychotherapy.

Participants were randomly allocated (ratio, 1:1) to either the treatment (ABMT) or control (sham ABMT) group by an independent researcher using block randomisation, with the sequence generated by an online tool (www. sealedenvelope.com) and a block size of 4 (Figure). The schedule, environment, and equipment used for both groups were identical. Participants, the research, and the assessor were blinded to the group allocation.

The treatment group received 4 weekly sessions of ABMT in addition to usual psychiatric care (consultations with psychiatrists and/or administration of psychiatric medication), whereas the control group received 4 weekly sessions of sham ABMT in addition to usual psychiatric care. Participants were assessed before training and then received training within 1 week and were reassessed 1 week after completion of the training.

\section{Attentional Bias Modification Training}

Sessions were conducted in a quiet and isolated room and administered individually using a desktop computer through the E-prime 2.0 Professional software (Psychology Software Tools, Pittsburgh, PA, USA). All participants received the same set of stimuli in the same order. ABMT was modified from the visual probe procedure ${ }^{14}$ and was based on studies that reported positive results. ${ }^{10,22}$

We used pictures of facial expressions as stimuli instead of words, because GAD patients have been shown to exhibit attentional bias towards facial expressions with negative valence, ${ }^{11}$ and because the perception of facial expressions is less bound by cultural factors. ${ }^{23}$ The pictures consisted of facial expressions adopted from the Japanese and Caucasian Facial Expressions of Emotion collection. ${ }^{24}$ The emotionality of pictures has been validated in both Western and Asian cultures. ${ }^{24}$ Each picture measured 5.7 $\times 4 \mathrm{~cm}$. The participant's face was approximately $65 \mathrm{~cm}$ from the screen. This gave a $4.5^{\circ}$ viewing angle between the centres of the two pictures. One of the pictures was a threatening facial expression (anger, disgust or fear), ${ }^{25}$ whereas the other was an expression of neutral emotion. The positions of pictures were randomised.

In each trial, a cross was shown in the centre of the computer screen for $500 \mathrm{~ms}$. The cross then disappeared and two pictures (one above and one below the centre) appeared for $500 \mathrm{~ms}$. After $500 \mathrm{~ms}$, a probe (an arrow pointing to either left ' $<$ ' or right ' $>$ ') replaced the picture. The probe was programmed to always appear in the position preceded by a neutral face. Participants were asked to respond to the direction of the probe as quickly as possible by clicking either the left or right button coinciding with the direction of the probe. Behavioural responses and reaction times were recorded.

To conceal the intervention mechanism, $10 \%$ of the trials were neutral trials, in which both pictures presented were neutral faces and the probe that followed was random in its position. ${ }^{10}$ 


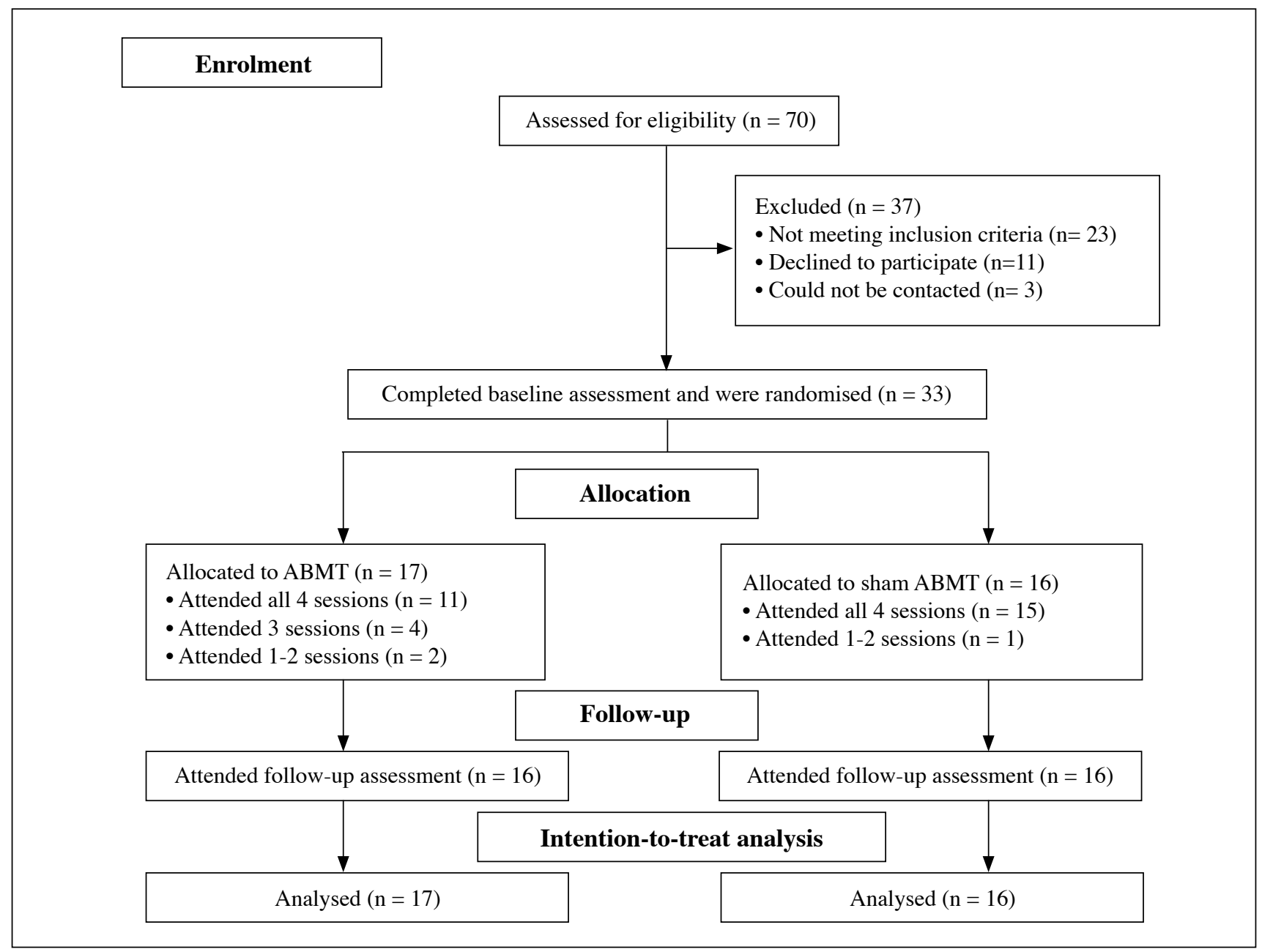

Figure. CONSORT flow diagram.

Each session had 360 trials and lasted about 15 minutes. There was a break after every 120 trials to prevent fatigue. Each participant was trained with a total of 1296 active trials, which is comparable to the 1280 trials administered in the Amir et al study, ${ }^{10}$ although our study used a more clinically feasible weekly schedule.

\section{Sham Attentional Bias Modification Training}

The sham ABMT procedure was essentially the same as the ABMT procedure, except that the position of the probes was not linked to pictures of a particular emotionality. The key treatment element was thus lacking.

\section{Outcome Measures}

Anxiety was measured using the Hamilton Anxiety Rating Scale (HAM-A) ${ }^{25}$ and the State-Trait Anxiety Inventory trait anxiety subscale (STAI-T) ${ }^{26}$ The HAM-A is a 14 -item, clinician-rated scale and has high internal consistency and good inter-rater and intra-rater reliability and sensitivity to change. ${ }^{27}$ The STAI-T consists of 20 self-rated items and has excellent test-retest stability. It is one of the most widely used self-reported anxiety measurements. ${ }^{28}$ The Chinese version has been validated locally. ${ }^{29}$

Pathological worry was measured using the 16-item Penn State Worry Questionnaire (PSWQ). ${ }^{30}$ It has good internal consistency, test-retest reliability, and sensitivity to change ${ }^{31}$ and has been validated in a Chinese population. ${ }^{32}$

Attentional bias towards threatening or neutral stimuli was measured using the bias score. ${ }^{33}$ This score provides a simplified representation of differences in participants' probe response times when presented with different combinations of stimulus and probe positions while performing the visual probe procedure. Its formula is:

$$
[(\text { UPLT-UPUT) }+(\text { LPUT-LPLT) }] / 2 \text {, }
$$

where UPLT (upper probe lower threat) refers to the participants' probe response time when the probe appeared in the upper position and the threatening stimulus appeared in the lower position. UPUT (upper probe upper threat), LPUT (lower probe upper threat), and LPLT (lower probe lower threat) can be understood similarly. Trials with extreme response times (defined as $<200 \mathrm{~ms}$ or $>1000 \mathrm{~ms}$ ) were excluded from calculation. A bias score of 0 indicates that the positions of the probe and threatening stimuli had no effect on probe response time (ie, no attentional bias). 
Positive scores indicate attentional bias towards threatening stimuli that the participant responded faster when the probe appeared in the position previously occupied by a threatening stimulus. Negative sores indicate opposite. A Cedrus RB450 response pad (Cedrus Corporation, San Pedro, CA, USA) was used to increase the accuracy of response time registration.

\section{Statistical Analysis}

Sample size estimation was based on the Amir et al. study. ${ }^{10}$ The pooled standard deviation of the HAM-A score of subjects was 7 . The difference in the mean outcome between the treatment and control groups was 6.1. To achieve an $\alpha$ of $5 \%$ and a power of 0.8 , we estimated that 20 patients were needed in each group.

Statistical analysis was performed using SPSS (Mac OS version 21; IBM Corp, Armonk [NY], US). Independent sample t-tests were used to compare baseline differences in primary and secondary outcome measures between groups. Repeated-measures ANOVA was used to test time (pretraining vs post-training) $\mathrm{x}$ group (treatment vs control) effects. Outcome measures were analysed according to the intention-to-treat principle. Missing data were handled using the baseline observation carried forward method. Missing baseline data were imputed using the mean value of the group. A separate per-protocol analysis was carried out.

\section{Results}

Of 70 referred patients, 33 met the inclusion criteria, consented to participate, and were allocated to the treatment $(n=17)$ or control $(n=16)$ group (Table 1$) .88 \%$ of participants in the treatment group and $94 \%$ in the control

Table 1. Demographic and clinical data of participants at baseline*

\begin{tabular}{|lcc|}
\hline Age, years & Treatment (n= 17) & Control (n= 16) \\
\hline Female sex & $44.3 \pm 12.9$ & $49.4 \pm 9.5$ \\
\hline Duration of illness, years & $9(53)$ & $9(56)$ \\
\hline Education, years & $10.4 \pm 9.0$ & $12.9 \pm 8.8$ \\
\hline Currently employed & $12 \pm 4.5$ & $11.8 \pm 4.6$ \\
\hline Receiving antidepressant or anxiolytic medication & $6(35)$ & $8(50)$ \\
\hline Change of medication during study period & $15(88)$ & $13(81)$ \\
\hline Co-morbid depression & $3(18)$ & $1(6)$ \\
\hline
\end{tabular}

* Data are presented as mean \pm standard deviation or No. (\%) of participants

Table 2. Comparison of outcome measures between groups using intention-to-treat analysis with repeated measures ANOVA*

\begin{tabular}{|c|c|c|c|c|c|c|}
\hline \multirow[t]{2}{*}{ Outcome measure } & \multirow[t]{2}{*}{$\begin{array}{l}\text { Treatment } \\
(\mathbf{n}=17)\end{array}$} & \multirow[t]{2}{*}{$\begin{array}{l}\text { Control } \\
(\mathbf{n}=15)\end{array}$} & \multicolumn{2}{|c|}{ Time } & \multicolumn{2}{|c|}{$\begin{array}{l}\text { Time } x \text { group } \\
\text { interaction }\end{array}$} \\
\hline & & & $\boldsymbol{F}$ & P value & $\boldsymbol{F}$ & P value \\
\hline $\begin{array}{l}\text { Hamilton Anxiety Rating Scale score } \\
\text { Pre-training assessment } \\
\text { Post-training assessment }\end{array}$ & $\begin{array}{l}20.8 \pm 6.4 \\
18.5 \pm 7.2\end{array}$ & $\begin{array}{l}23.3 \pm 11.1 \\
19.6 \pm 9.4\end{array}$ & 6.6 & 0.009 & 0.3 & 0.51 \\
\hline $\begin{array}{l}\text { State Trait Anxiety Inventory - trait } \\
\text { anxiety subscale score } \\
\text { Pre-training assessment } \\
\text { Post-training assessment }\end{array}$ & $\begin{array}{l}57.7 \pm 11.4 \\
55.9 \pm 11.6\end{array}$ & $\begin{array}{l}57.1 \pm 7.6 \\
51.5 \pm 6.9\end{array}$ & 11.7 & 0.002 & 3.2 & 0.09 \\
\hline $\begin{array}{l}\text { Penn State Worry Questionnaire score } \\
\text { Pre-training assessment } \\
\text { Post-training assessment }\end{array}$ & $\begin{array}{l}64.4 \pm 10.9 \\
58.9 \pm 11.6\end{array}$ & $\begin{array}{l}57.6 \pm 11.4 \\
56.5 \pm 11.6\end{array}$ & 6.7 & 0.015 & 2.1 & 0.16 \\
\hline $\begin{array}{l}\text { Bias score } \\
\text { Pre-training assessment } \\
\text { Post-training assessment }\end{array}$ & $\begin{array}{l}-3.5 \pm 10.7 \\
-6.7 \pm 8.5\end{array}$ & $\begin{array}{l}-0.7 \pm 18.3 \\
-0.8 \pm 11.0\end{array}$ & 0.4 & 0.53 & 0.4 & 0.54 \\
\hline
\end{tabular}

Data are presented as mean \pm standard deviation 
group attended at least 3 out of 4 training sessions. One participant from the treatment group dropped out after two sessions; missing data were dealt with using the baseline observation carried forward method. One participant from the control group refused to complete the self-reported questionnaires because she felt overwhelmed; missing scores were imputed by the mean scores of the same group.

For hypothesis 1, the two groups were comparable in terms of baseline scores of HAM-A $(\mathrm{t}=0.78$, $\mathrm{df}=23.7$, $\mathrm{p}=0.44)$, STAI-T $(t=0.17, d f=28.0, \mathrm{p}=0.87)$, and PSWQ $(t=1.76, d f=30, \mathrm{p}=0.09)$. The Levene test indicated unequal variances in the HAM-A $(F=4.4, \mathrm{p}=.045)$ and STAI-T $(F=4.6, \mathrm{p}=0.04)$ scores from the two groups. Repeated-measures ANOVA yielded a significant effect of time on HAM-A score $(F(1,31)=7.9, \mathrm{p}=0.009)$, STAI-T score $(F(1,31)=11.0, \mathrm{p}=0.002)$, and PSWQ score $(F(1,31)$ $=7.0, \mathrm{p}=0.013)$, but no significant time $\mathrm{x}$ group interaction on the respective scores $(F(1,31)=0.5, \mathrm{p}=0.51),(F(1,31)$ $=2.7, \mathrm{p}=0.1)$, and $(F(1,31)=2.3, \mathrm{p}=0.14)$ [Table 2]. Participants in the treatment group reported a decrease in anxiety and worry, although this decrease was not greater in magnitude than that of participants in the control group.

For hypothesis 2 , the two groups were comparable in terms of baseline bias score $(t=-0.52, d f=31, \mathrm{p}=0.60)$. Repeated-measures ANOVA yielded no significant effect of time or time $\mathrm{x}$ group interaction on bias score $(F(1,31)=$ $0.39, \mathrm{p}=0.54$, Table 2).

For hypothesis 3 , the change in bias score was not correlated to changes in scores of HAM-A $(r=0.03$, $\mathrm{p}=0.87)$, STAI-T $(r=0.07, \mathrm{p}=0.69)$, and PSWQ $(r=0.29$, $\mathrm{p}=0.11)$.

11 participants from the treatment group and 14 participants from the control group completed all sessions and assessments. Repeated measures ANOVA yielded a significant effect of time on scores of HAM-A $(F(1,23)=$ $6.5, \mathrm{p}=0.02)$, STAI-T $(F(1,23)=9.5, \mathrm{p}<0.01)$, and PWSQ $(F(1,23)=4.3, \mathrm{p}=0.049)$ but no significant time $\mathrm{x}$ group interactions. There was no significant effect of time or time $\mathrm{x}$ group interaction on bias score.

One participant from the treatment group complained of increased floaters and blurring of vision after one session. She had been treated for retinal detachment 10 years earlier; consultation to an ophthalmologist showed no retinal pathology or significant deterioration in vision. 31 of the 33 participants rated the treatment as an acceptable form of therapy.

\section{Discussion}

Both ABMT and sham ABMT reported a significant reduction in levels of anxiety and worry after intervention, but the reduction was not greater after ABMT than sham ABMT. The reduction in symptoms could not be explained by the hypothesised mechanism of ABMT. In addition, ABMT did not induce a significant attentional bias in favour of nonthreatening stimuli over threatening stimuli. Subject factors and intervention factors may have contributed to the results.

\section{Subject factors}

The outcome of psychological treatment is affected by the characteristics of the patients. ${ }^{34}$ Our participants were recruited from specialist psychiatric clinics; most still had clinically significant symptoms despite medication treatment, suggesting more severe and resistant illness.

In addition, antidepressants may have had affected the results. Change in attention processing is one of the hypothesised mediators of the treatment effects of antidepressant medication. ${ }^{36} \mathrm{~A}$ short course of citalopram reduces healthy subjects' attentional bias towards fearful faces. ${ }^{35}$ It is possible that this treatment pathway had already been saturated, as reflected by the lack of attentional bias towards threat-related stimuli in our sample. If so, ABMT would not have conferred additional change.

The lack of attentional bias towards threatening stimuli at baseline might also suggest heterogeneity within our GAD patients in terms of threat-seeking/threatavoidance tendencies, although this hypothesis needs to be tested. A meta-analysis reported that there is no moderation effect of pre-existing attentional bias on treatment outcome, and that young age is a moderating factor. ${ }^{19}$ Our participants were relatively older than those in other studies and may have contributed to the lack of change in attentional bias.

\section{Intervention factors}

The most effective ABMT protocol remains unknown. There is considerable variation in the specifications of protocols among studies (eg, choice of stimuli and treatment schedule). For stimuli, we chose threatening facial expressions over words with threatening meanings because the former is a more primitive source of threat and are relatively universal across different cultures. The Japanese and Caucasian Facial Expressions of Emotion collection has been used in researches relating to attentional bias and attentional bias modification..$^{22,37,38}$ Nonetheless, individual differences in the registration of the emotional valence of particular stimuli may affect the outcome of a particular ABMT protocol. A review suggested that future ABMT studies should consider designs that promote registration of stimulus valence. ${ }^{39}$

In our study, the lack of effect of ABMT could also be related to the low-intensity schedule. The dose-response relationship of the effect of ABMT has not been established in previous studies, in which treatment schedules were more frequent than once per week. We could only offer a weekly schedule because of the consideration of treatment adherence. Nonetheless, the total number of training trials was on par with that in previous studies that yielded positive results. The dose-response relationship needs to be achieved by larger studies with treatment arms that have different treatment intensities.

\section{Non-specific effect of psychological therapies}

In our study, the only difference between the treatment and control groups was the positioning of the probe in the space preceded by the neutral stimuli as opposed to 
random positioning. The comparable outcome between the two groups indicated a non-specific effect shared by the two groups, which has been demonstrated in a study on ABMT. ${ }^{40}$ The non-specific effect can still be significant even in a computerised approach with a relatively low amount of clinical contact.

\section{Limitations}

The final number of participants recruited fell short of the target, and the study may have been underpowered and increased type II error. However, our results did not indicate a trend of differential change between the treatment and control groups, and therefore insufficient statistical power alone may not account for the negative results. In addition, the follow-up period was short, with only one measurement time point at 1-week post intervention. There may be concerns relating to the delayed effect that changes in attentional bias brought about by ABMT takes time to reflect on a symptom level. However, no changes in attentional bias were detected in our participants and thus no delayed effect was expected.

\section{Conclusion}

There was non-specific treatment effect of ABMT on anxiety and worry in GAD patients. However, specific clinical efficacy of ABMT was not demonstrated. Our results do not support the use of ABMT as a treatment option for GAD patients. The three hypotheses are not supported. The small sample size and short duration of treatment may have rendered the results inconclusive. Further randomised controlled trials are needed to confirm the efficacy of ABMT demonstrated in previous studies. There is a large knowledge gap between ABMT theories and its application in a clinical setting.

\section{Acknowledgement}

We thank Ms Daisy Cheung for her help with proofreading.

\section{Declaration}

This study did not receive any grant from funding agencies in the public, commercial, or not-for-profit sectors. Suzanne Ho-wai So has received funding from the Croucher Foundation Startup Fund. This study was conducted for the partial fulfilment of the Fellowship examination of the Hong Kong College of Psychiatrists of Steven Wai Ho Chau. The authors have no conflicts of interest to disclose.

\section{References}

1. American Psychiatric Association. Diagnostic and Statistical Manual of Mental Disorders. 5th ed. Washington, DC; 2013.

2. Lee S, Tsang A, Chui H, Kwok K, Cheung E. A community epidemiological survey of generalized anxiety disorder in Hong Kong. Community Ment Health J 2007;43:305-19. Crossref

3. Hoffman DL, Dukes EM, Wittchen HU. Human and economic burden of generalized anxiety disorder. Depress Anxiety 2008;25:72-90. Crossref

4. National Institute for Health and Care Excellence (NICE). Generalised Anxiety Disorder and Panic Disorder in Adults: Management. London: NICE; 2011.

5. Baldwin D, Woods R, Lawson R, Taylor D. Efficacy of drug treatments for generalised anxiety disorder: systematic review and meta-analysis. BMJ 2011;342:d1199. Crossref

6. Beck AT, Clark DA. An information processing model of anxiety: automatic and strategic processes. Behav Res Ther 1997;35:4958. Crossref

7. Kim YW, Lee SH, Choi TK, Suh SY, Kim B, Kim CM, et al. Effectiveness of mindfulness-based cognitive therapy as an adjuvant to pharmacotherapy in patients with panic disorder or generalized anxiety disorder. Depress Anxiety 2009;26:601-6. Crossref

8. Mitte K. Meta-analysis of cognitive-behavioral treatments for generalized anxiety disorder: a comparison with pharmacotherapy. Psychol Bull 2005;131:785-95. Crossref

9. MacLeod C, Mathews A. Cognitive bias modification approaches to anxiety. Annu Rev Clin Psychol 2012;8:189-217. Crossref

10. Amir N, Beard C, Burns M, Bomyea J. Attention modification program in individuals with generalized anxiety disorder. J Abnorm Psychol 2009;118:28-33. Crossref

11. Bradley BP, Mogg K, White J, Groom C, de Bono J. Attentional bias for emotional faces in generalized anxiety disorder. Br J Clin Psychol 1999;38:267-78. Crossref

12. Amir N, Weber G, Beard C, Bomyea J, Taylor CT. The effect of a single-session attention modification program on response to a publicspeaking challenge in socially anxious individuals. J Abnorm Psychol 2008;117:860-8. Crossref

13. Dandeneau SD, Baldwin MW, Baccus JR, Sakellaropoulo M, Pruessner JC. Cutting stress off at the pass: reducing vigilance and responsiveness to social threat by manipulating attention. J Pers Soc Psychol 2007;93:651-66. Crossref

14. Browning M, Holmes EA, Harmer CJ. The modification of attentional bias to emotional information: a review of the techniques, mechanisms, and relevance to emotional disorders. Cogn Affect Behav Neurosci 2010;10:8-20. Crossref

15. Posner MI, Petersen SE. The attention system of the human brain. Annu Rev Neurosci 1990;13:25-42. Crossref

16. Browning M, Holmes EA, Murphy SE, Goodwin GM, Harmer CJ. Lateral prefrontal cortex mediates the cognitive modification of attentional bias. Biol Psychiatry 2010;67:919-25. Crossref

17. Beard C, Sawyer AT, Hofmann SG. Efficacy of attention bias modification using threat and appetitive stimuli: a meta-analytic review. Behav Ther 2012;43:724-40. Crossref

18. Hallion LS, Ruscio AM. A meta-analysis of the effect of cognitive bias modification on anxiety and depression. Psychol Bull 2011;137:94058. Crossref

19. Mogoaşe C, David D, Koster EH. Clinical efficacy of attentional bias modification procedures: an updated meta-analysis. J Clin Psychol 2014;70:1133-57. Crossref

20. Linetzky M, Pergamin-Hight L, Pine DS, Bar-Haim Y. Quantitative evaluation of the clinical efficacy of attention bias modification treatment for anxiety disorders. Depress Anxiety 2015;32:38391. Crossref

21. First MB, Spitzer RL, Gibbon M, Williams JB. Structured Clinical Interview for DSM-IV-TR Axis I Disorders, Research Version, Patient Edition. (SCID-I/P) New York: Biometrics Research, New York State Psychiatric Institute; 2002.

22. Rozenman M, Weersing VR, Amir N. A case series of attention modification in clinically anxious youths. Behav Res Ther 2011;49:32430. Crossref

23. Elfenbein HA, Ambady N. On the universality and cultural specificity of emotion recognition: a meta-analysis. Psychol Bull 2002;128:20335. Crossref

24. Matsumoto D, Ekman P. American-Japanese cultural differences in intensity ratings of facial expressions of emotion. Motiv Emot 1989;13:143-57. Crossref 
25. Hamilton M. Diagnosis and rating of anxiety. Br J Psychiatry 1969;3:76-9.

26. Spielberger CD. Manual for the State-Trait Anxiety Inventory (Form Y). Consulting Psychologists Press; 1983.

27. Maier W, Buller R, Philipp M, Heuser I. The Hamilton Anxiety Scale: reliability, validity and sensitivity to change in anxiety and depressive disorders. J Affect Disord 1988;14:61-8. Crossref

28. Piotrowski C. The status of the Beck Anxiety Inventory in contemporary research. Psychol Rep 1999;85:261-2. Crossref

29. Shek DT. The Chinese version of the State-Trait Anxiety Inventory: its relationship to different measures of psychological well-being. J Clin Psychol 1993;49:349-58. Crossref

30. Meyer TJ, Miller ML, Metzger RL, Borkovec TD. Development and validation of the Penn State Worry Questionnaire. Behav Res Ther 1990;28:487-95. Crossref

31. Dear BF, Titov N, Sunderland M, McMillan D, Anderson T, Lorian $\mathrm{C}$, et al. Psychometric comparison of the generalized anxiety disorder scale-7 and the Penn State Worry Questionnaire for measuring response during treatment of generalised anxiety disorder. Cogn Behav Ther 2011;40:216-27. Crossref

32. Zhong J, Wang C, Li J, Liu J.Penn State Worry Questionnaire: structure and psychometric properties of the Chinese version. J Zhejiang Univ Sci B 2009;10:211-8. Crossref

33. MacLeod C, Mathews A, Tata P. Attentional bias in emotional disorders. J Abnorm Psychol 1986;95:15-20. Crossref
34. Beutler LE, Mohr DC, Grawe K, Engle D, MacDonald R. Looking for differential treatment effects: cross-cultural predictors of differential psychotherapy efficacy. J Psychother Integr 1991;1:121-41. Crossref

35. Murphy SE, Yiend J, Lester KJ, Cowen PJ, Harmer CJ. Short-term serotonergic but not noradrenergic antidepressant administration reduces attentional vigilance to threat in healthy volunteers. Int $\mathbf{J}$ Neuropsychopharmacol 2009;12:169-79. Crossref

36. Harmer CJ, Goodwin GM, Cowen PJ. Why do antidepressants take so long to work? A cognitive neuropsychological model of antidepressant drug action. Br J Psychiatry 2009;195:102-8. Crossref

37. Bar-Haim Y, Lamy D, Pergamin L, Bakermans-Kranenburg MJ, van IJzendoorn $\mathrm{MH}$. Threat-related attentional bias in anxious and nonanxious individuals: a meta-analytic study. Psychol Bull 2007;133:1-24. Crossref

38. Carlbring P, Apelstrand M, Sehlin H, Amir N, Rousseau A, Hofmann $\mathrm{SG}$, et al. Internet-delivered attention bias modification training in individuals with social anxiety disorder--a double blind randomized controlled trial. BMC Psychiatry 2012;12:66. Crossref

39. MacLeod C, Clarke PJ. The attentional bias modification approach to anxiety intervention. Clin Psychol Sci 2015;3:58-78. Crossref

40. Shechner T, Rimon-Chakir A, Britton JC, Lotan D, Apter A, Bliese $\mathrm{PD}$, et al. Attention bias modification treatment augmenting effects on cognitive behavioral therapy in children with anxiety: randomized controlled trial. J Am Acad Child Adolesc Psychiatry 2014;53:6171. Crossref 